\title{
Sich rechtzeitig der Herausforderung stellen
}

\author{
Marcus Lippe und Ulrike Lessig
}

Die "ambulanten dienste e. $V$. in Berlin sind mit rund 560 Angestellten ein großer Anbieter von Assistenzdienstleistungen für Menschen mit Behinderung. Die Organisation reagierte früh auf die absehbare Einführung des Persönlichen Budgets: mit einer Modularisierung ihrer Angebote und mit einem neuen Angebot, dem »Splittingmodell«.

Es ist eine alte Forderung der Behindertenbewegung, dass das Geld in die Hände der behinderten Menschen gehört. Von Anfang an war es für die im Jahre 1981 gegründeten »ambulanten dienste e. V. $(\mathrm{ad})$ « in Berlin ein zentrales Anliegen, behinderten Menschen ein selbstbestimmtes und selbst verantwortetes Leben zu ermöglichen. Deshalb gab es die Praxis, das Geld der Leistungsträger, das an die "ambulanten dienste « für die bewilligte Leistung überwiesen wurde, weiter an die Assistenznehmerinnen und Assistenznehmer zu überwiesen, um es bei ihnen wieder in Rechnung zu stellen.

Was wie eine umständliche Regelung wirkt, hat den Kunden und Kundinnen ein anderes Gefühl als Assistenznehmerinnen und Assistenznehmer gegeben, und ihnen natürlich die Freiheit ermöglichte, das Geld auch bei anderen Anbietern auszugeben. Mit der zunehmenden Bürokratisierung der ambulanten Versorgung, insbesondere der Einführung der Pflegeversicherung war das nicht mehr möglich.

Inzwischen beschäftigen die »ambulante dienste « in Berlin zirka 560 Angestellte und versorgen über 100 körperlich behinderte Assistenznehmerinnen und Assistenznehmer, die überwiegend einen Assistenzbedarf von mehr als fünf Stunden täglich benötigen, mit rund 40.000 Assistenzstunden im Monat. Der gemeinnützige Verein ist damit einer der größten Dienstleister in diesem Bereich in der Bundesrepublik. Es amtiert ein dreiköpfiger Vorstand. Die zweimal jährlich zusammentretende Mitgliederversammlung ist das höchste Beschlussorgan des Vereins. Behinderte Menschen genießen besondere Einflussrechte und arbeiten in Verein und Organisation an maßgeblicher Stelle mit. Ein Vorstandsmitglied ist selbst Assistenznehmer. Die Vertretung der Assistenznehmerinnen und Assistenznehmer hat innerhalb des Vereins und innerhalb des Betriebes weitgehende Informations-, Mitsprache- und Vetorechte. Zudem engagiert sich der Verein über Themen der Selbstbestimmung und Assistenz hinaus, allgemein behindertenpolitisch. Die 160 Mitglieder sind die entscheidenden Träger der behindertenpolitischen Grundsätze.

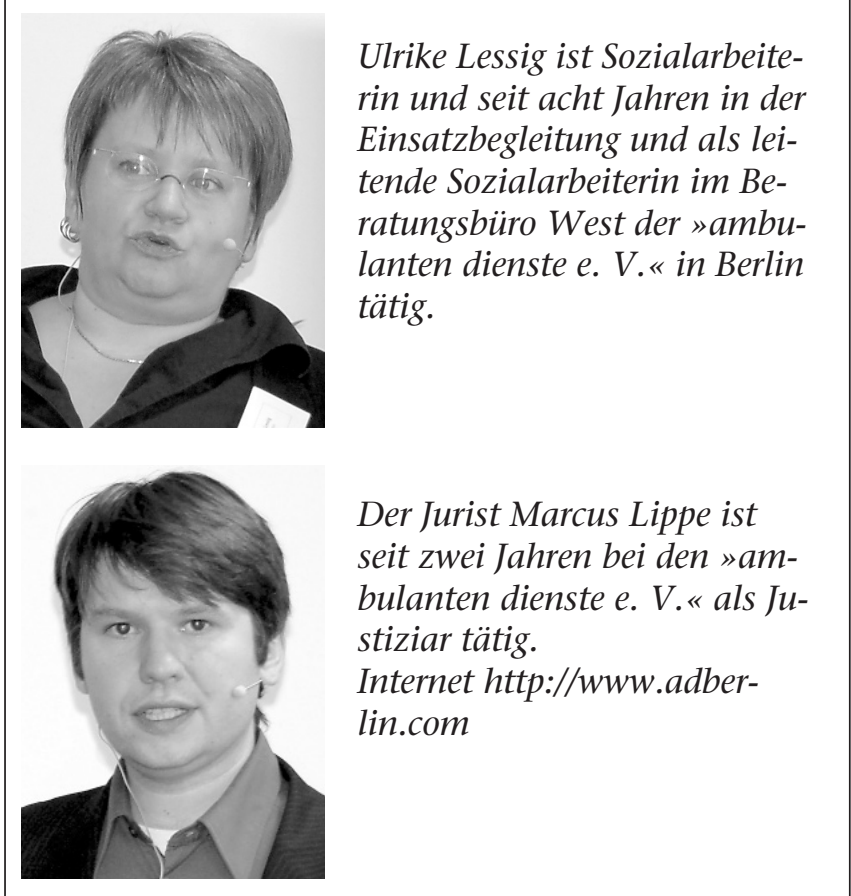

Im Hinblick auf die alte behindertenpolitische Forderung wurde die Einführung des trägerübergreifenden Persönlichen Budgets in der Berliner Modellregion Bezirk Friedrichshain-Kreuzberg seit 2005 begrüßt, auch wenn dies sowohl den Verlust von Kunden, als auch die Umstellung des Angebotes auf die neuen Budgetnehmerinnen und Budgetnehmer bedeutete.

\section{Veränderungen durch das Persönliche Budget}

Beide Punkte sind Konsequenzen der grundsätzlichen Veränderungen, die mit der Einführung des Persönlichen Budgets einhergeht. In der Wahrnehmung der »ambulanten dienste« waren dabei zentral:

- Das Dreiecksverhältnisses zwischen Leistungsträger, Leistungsempfänger und Leistungserbringer wird aufgelöst. Diese Auflösung bedeutet: Es gibt zwischen Leistungsträger und Leistungserbringer keine Verhandlungen mehr über die Erbringung der jeweiligen Leistung.

- Zwischen dem Leistungserbringer und dem Budgetnehmer oder der Budgetnehmerin muss ein zivilrechtlicher Vertrag geschlossen werden. Daraus folgt ein Weniger 
an Planungssicherheit für den Leistungserbringer, weil die zeitliche Bewilligung - meistens des Sozialamtes nicht mehr die unmittelbare Vertragsgrundlage ist, sondern allein der privatrechtliche Vertrag zwischen den »ambulanten diensten « und dem Budgetnehmer oder der Budgetnehmerin.

- Die Rolle der Budgetnehmer und Budgetnehmerinnen als Kunden wird gestärkt, wobei gleichzeitig Selbstbestimmung und Selbstverantwortlichkeit für die Organisation der Assistenz zunimmt.

Diese Einschätzung basierte nicht nur auf der rechtlichen Ausgestaltung des Persönlichen Budgets, sondern auch auf Äußerungen und Wünschen von Assistenznehmerinnen und Assistenznehmern. Einige von ihnen hatten nur darauf gewartet, endlich eine gesetzliche Grundlage zu bekommen, um ihre Versorgung selbst zu organisieren. Diese Assistenznehmerinnen und Assistenznehmer hatten schon als Kunden der »ambulanten dienste« die Möglichkeit, ihren eigenen Einsatz weitgehend autonom zu gestalten. Es war lediglich die Abrechnung mit den Leistungsträgern, die von einer Organisation gemacht werden musste, und somit die Assistenznehmerinnen und Assistenznehmer zwangen, sich zu binden.

Bei den »ambulanten diensten « hat die Mischung von selbstorganisierten Einsätzen und Einsätzen, die viel Begleitung benötigten, ein Solidarsystem gebildet, das durch die Abwanderung der einen Seite den Betrieb in eine schwierige Lage gebracht hat. Gleichzeitig fragten Budgetnehmer und Budgetnehmerinnen als neue Kunden nach einzelnen Bausteinen unserer bisherigen Leistungen.

\section{Die organisatorische Umsetzung - ein Dreisprung}

Deshalb wurde innerhalb der »ambulanten dienste eine Arbeitsgruppe gegründet, die sich aus der Geschäftsführerin, einem Mitglied des Vorstandes, dem Justiziar und weiteren Mitarbeiterinnen aus der Leitungsebene zu- sammensetzt. Hier fand eine intensiv Auseinandersetzung mit den neuen Möglichkeiten statt, insbesondere mit Frage, wie die »ambulanten dienste « auf die Nachfrage von zukünftigem Budgetnehmern und Budgetnehmerinnen nach einzelnen Leistungen reagieren könnte.

1. Es wurde in drei Schritten vorgegangen. Zuerst - setzte sich die Arbeitsgruppe darüber auseinander, welche Leistungen im Einzelnen erbracht werden. Bis dahin wurden die einzelnen Tätigkeiten als Teil der Assistenzleistungen betrachtet, ohne dass eine Differenzierung vorgenommen wurde. Dabei wurden folgende Leistungen unterschieden:
- persönliche Assistenz vor Ort durch die angestellten Assistenten und Assistentinnen

- Beratung durch Sozialarbeiter und Sozialarbeiterinnen, Pflegefachkräfte und Juristen, bei sozialrechtlichen, psychosozialen, pflegerischen und das Personal betreffenden Fragen

- Begleitungs- und Unterstützungsleistungen rund um die Assistenz

- einsatzbezogene Qualifizierung der Assistenten unD Assistentinnen

2 Im nächsten Schritt wurden Überlegungen ange• stellt, wie die einzelnen Leistungen im Rahmen des Persönlichen Budgets als Einzelleistung erbracht werden können und welche Veränderungen dafür nötig sind. Dabei wurden folgende Leistungen als budgetfähig erachtet:

- persönliche Assistenz auch in Zusammenarbeit mit Assistenten und Assistentinnen, die durch den Budgetnehmer gestellt werden (»Splittingmodell«)

- Beratung durch Sozialarbeiterinnen und Sozialarbeiter sowie einen Juristen bei Bewilligung und Verwendung des Budgets

- Begleitung und Unterstützung bei Einsätzen, die von dem Budgetnehmer selbst organisiert werden

Die »ambulanten dienste e. V.« in Berlin betreiben einen Assistenzdienst für behinderte Menschen. Im Hinblick auf die Einführung des Persönlichen Budgets wurde dort das »Splittingmodell « entwickelt. Dabei organisiert der behinderte Budgetnehmer eine bestimmte Zeit, beispielsweise das Wochenende, seinen Assistenzbedarf selbst durch Freunde, Nachbarn oder andere Helfer. Die restliche Zeit wird, vertraglich geregelt, durch die angestellten Assistenzkräfte der »ambulanten dienste « abgedeckt.

Internet http://www.adberlin.com

- Qualifizierung von Assistenten und Assistentinnen, die durch den Budgetnehmer privat angestellt werden zu den Themen: Transfertechnik, Rollstuhltraining, Pflege, Nähe und Distanz, Hygiene und Arbeitsschutz, ErsteHilfe-Kurs

2. Nachdem nun deutlich wurde, welche Einzelleis3. tungen im Rahmen des Persönlichen Budgets von den »ambulanten diensten « erbracht werde konnten, er- 
folgte in einem letzten Schritt die Umsetzung in rechtlich abgesicherte und betriebswirtschaftlich durchkalkulierte Leistungsangebote. Dabei rückten zum einen die Frage der Verpreislichung der einzelnen Leistungen zum anderen die Vertragsgestaltung, insbesondere im Rahmen der weiterhin zentralen Leistung der persönlichen Assistenz, in den Vordergrund.

\section{Verpreislichung und Vertragsgestaltung}

Die Verpreislichung der einzelnen Leistungen gestaltete sich unterschiedlich schwierig. Während für die persönliche Assistenz einfach auf die vorhandenen Kostensätze zurückgegriffen werden konnte, wurden für die anderen Leistungen zunächst die Personal- und Sachkosten ermittelt. Aufgrund dieser Kalkulation fand dann die eigentliche Preisermittlung statt.

Bei der Vertragsgestaltung für die persönliche Assistenz wurden gegenüber den Verträgen im Rahmen des sozialrechtlichen Dreiecksverhältnisses in den Bereichen Kündigung, Stornierung, Datenschutz, Mitwirkungspflichten und Hinweispflichten Veränderungen notwendig. Hiefür waren zwei Gründe zentral:

Der ersten Grund ergibt sich aus der geringeren Planungssicherheit im Zusammenhang mit dem trägerübergreifenden Persönlichen Budget, von der die »ambulanten dienste« wegen ihrer stark am einzelnen Einsatz gebundenen Personals besonders betroffen sind. Der zweite Grund findet sich in der Einführung des Splittingmodells: Es mussten neuen Regelungen hinsichtlich des Kommunikationsflusses zwischen den Assistentinnen und Assistenten gefunden werden, die bei den »ambulanten diensten « angestellt sind und denjenigen Helfern, die von dem Budgetnehmer oder der Budgetnehmerin direkt gestellt werden. Dabei mussten auch Regelungen zu den Aspekten Pflegehaftungsrisiken und Verantwortlichkeiten im Allgemeinen gefunden werden.

\section{Veränderte Begleitung}

Aber die Einführung des Persönlichen Budgets führte nicht nur zu einer Veränderung auf der organisatorischen Ebene - auch auf die Sozialarbeiterinnen und Sozialarbeiter kamen neue Anforderungen zu. Deren Aufgaben waren bisher die Begleitung innerhalb der Assistenz bei der Antragstellung, der Teamkoordination der Assistentinnen und Assistenten sowie bei der Ermittlung des Fortbildungsbedarfs. Gleichzeitig waren sie Ansprechpartner für Assistenznehmerinnen und Assistenznehmer sowie für Assistentinnen und Assistenten. Das Splittingmodell setzt eine vermehrte Kommunikation mit den Budgetnehmern und Budgetnehmerinnen voraus. Zwei Teams müssen gemeinsam koordiniert werden und auch an einem gemeinsamen Arbeitsplatz arbeiten. Dafür nehmen alle Assistentinnen und Assistenten an den Teamsitzungen teil, um pflegerelevante Themen zu besprechen, und Wünsche der Budgetnehmerinnen und Budgetnehmer zeitgleich zu hören, und die Kommunikation zwischen beiden Teams zu fördern.

\section{Die Situation heute}

Inzwischen haben die »ambulanten dienste« fünf Budgetnehmerinnen und Budgetnehmer, die ihre Assistenz im Splittingmodell organisieren. Alle waren vorher Assistenznehmerinnen und Assistenznehmer bei den »ambulanten diensten «. Werden die zwei Budgetnehmer hinzugezählt, die die »ambulanten dienste « ganz verlassen haben, entstand mit der Einführung des trägerübergreifenden Persönlichen Budgets ein Assistenzstundenverlust von 1.650 bei 40.100 geleisteten Assistenzstunden im Monat insgesamt. Weiterhin werden in einem Fall auch die von der Budgetnehmerin selbst organisierten Assistentinnen von den »ambulanten diensten « begleitet, die Einsatzplanung übernommen und die Budgetnehmerin umfassend unterstützt. Ebenfalls fanden diverse Qualifikationen von Assistentinnen und Assistenten statt, die nicht bei den »ambulanten diensten « angestellt sind.

Lediglich bei der Budgetberatung wurden die Leistungsangebote bisher nicht angenommen. Die Gründe hierfür liegen einerseits im bereits vorhandenen Angebot in Berlin und andererseits darin, dass schon bei der Bedarfsermittlung eine solche Beratung nicht ausreichend Berücksichtigung findet. Die betroffenen Budgetnehmerinnen und Budgetnehmer sind deshalb dazu gezwungen, das Geld hierfür an anderer Stelle zu sparen, da es bei der Ermittlung der Budgethöhe nicht eingestellt wurde. Hinzu tritt hierbei noch, dass bereits auf der rechtlichen Ebene nur in Ausnahmefällen eine Überschreitung der Kosten im Vergleich zum vorher erbrachten Leistungsumfang möglich ist. Es kommt aber immer wieder vor, dass gerade am Anfang eines Budgets ein Mehraufwand an Begleitung und Unterstützung notwendig ist. Die Gewährung von Ausnahmen wird aber angesichts der leeren Kassen der Leistungsträger sehr zurückhaltend gehandhabt.

Ebenfalls auf der Negativseite zu verbuchen ist die Tatsache, dass sich erhoffte Verwaltungseinsparungen bisher nicht realisiert haben. Diese Hoffnung besteht, weil jetzt ja nur noch mit dem Budgetnehmer oder der Budgetnehmerin abgerechnet werden muss und nicht mit verschiedenen Leistungsträgern. Leider war eine Einbeziehung der Pflegekassen in das trägerübergreifende Persönliche Budget bisher bei den Kunden von uns nicht möglich, sodass sich das Budget bisher auf Leistungen des Landes Berlin meist als Hilfe zur Pflege durch die Sozialämter beschränkt. Daraus folgt, dass diese Leistungen zwar direkt mit den Budgetnehmerinnen und Budgetnehmer abgerechnet werden können, aber die Leistungen der Pflegekassen weiterhin im Dreiecksverhältnis verbleiben und entsprechend abgerechnet werden müssen.

Trotz dieser Schwierigkeiten, der neuen Anforderungen für die Mitarbeiterinnen und Mitarbeiter und eines Assistenzstundenverlusts bleibt das trägerübergreifende Persönliche Budget zumindest für einen Assistenzdienst wie »ambulante dienste e. V.« in Berlin handhabbar. Demgegenüber steht ein Mehr an Selbstbestimmung für die betroffenen Menschen. 